\title{
The Zitterbewegung of planets and Moon in the string gravity
}

\author{
Miroslav Pardy \\ Department of Physical Electronics \\ and \\ Laboratory of Plasma physics \\ Masaryk University \\ Kotlářská 2, 61137 Brno, Czech Republic \\ e-mail:pamir@physics.muni.cz
}

May 3, 2020

\begin{abstract}
The string model of gravitational force was proposed by author 40 years ago (Pardy, 1980; 1996). In this model the string forms the mediation of the gravitational interaction between two gravitating bodies. It reproduces the Newtonian results in the first-order approximation and it predicts in the higher-oder approximations the existence of oscillations of the massive bodies interacting by the string. In case of the Moon it can be easily verified by NASA laser measurements.
\end{abstract}

PACS numbers: 03.20, 03.40, 02.30.J

\section{Introduction}

It is well known from the history of physics that the problem of action-at-a-distance was for the first time seriously considered by Newton, in his letter to Bentley (Bentley, 1692; 1838), which is cited in "Principia Mathematica" (Newton, 1966) and analysed in the Stanford Encyclopedia of Philosophy (2006).

Instead of resolution of this problem Newton suggested the phenomenological theory of the gravitational force, where there exists no answer concerning the dynamics, or, the mechanism of action-at-a-distance. Newton himself was awared that it necessary exists some mediation of interaction between two bodies at the different points in space because he has written (Newton. 1966): "It is inconceivable, that inanimate brute matter, should without the mediation of something else which is not material, operate upon and affect other matter without mutual contact .. ". In other words, the crucial notion in the Newton speculation is the mediation between two bodies. Now, we can say that Newton considered the string model of gravity because string model of gravity is the natural result of Newton scientific intuition.

The problem of mediation of the gravitational force between two bodies 1 and 2 can be analysed by the way which forms the serious motivations for reconsidering the problem od action-at-a-distance. If we trasmit body 1 with mass $m$ from one point to other during very short time interval, then the gravitational force acting to the second body 2 with 
mass $M$ necessary changes. However, in case that the second body is far from the first one, then much time elapses before it receives the gravitational input. The question is, where is the gravitational perturbation when the first body after short motion is yet in rest and the second one has no information on the motion on the first body?. It is evident the gravitational input is between body 1 and 2 on the line or string connecting body 1 with the body 2 . The string is the Newton medium which transmits the gravitational force from one body to the other one.

It is well known that Newtonian theory is successful in its domain of validity, and general relativity is successful in accounting for the discrepancies between observed gravitational data and the Newtonian theory, as well as in resolving its problem of compatibility with special relativity. Nevertheless, Newton theory does not involve the string tension which is logical necessary as we have seen.

By analogy with the mechanical situation we will suppose the model where the atractive force between two bodies is transmitted as tension in the fictitious string connecting the one body with the another one. Then, the theoretical problem is to show that such model works and gives not only the old results but new results which cannot be derived from the original Newton law.

We will consider the string, the left end of which is fixed at the beginning of the coordinate system and mass $m$ is fixed on the right end of the string. The motion of the system string and the body with mass $m$ is the fundamental problem of the equations of the mathematical physics in case that the tension is linearly dependent on elongation (Tikhonov et al., 1977). We will show that it is possible to represent the Newton gravitational law by the string with the nonlinear tension in the string. Because of the strong nonlinearity of the problem the motion of the string and the body can be solved only approximately. In the following text, we will give the aproximative solution of the classical two-body problem and then we obtaine the string solution of this problem.

\section{The classical two-body problem}

Let us consider two bodies 1 and 2 with masses $M$ and $m$, where $M \gg m$. The body 1 is supposed to be fixed at the origin of the coordinate system and the body 2 is for the simplicity moving in the interval

$$
(R-\delta, R+\delta)
$$

where $\delta \ll R$, which corresponds to the motion of planets of our Sun system. The Newton law

$$
F=-\kappa \frac{M m}{r^{2}}
$$

can be obviously expressed in the interval (1) approximatelly as

$$
F \approx a \eta+b ; \quad(-\delta, \delta) \ni \eta
$$

where

$$
a=\frac{2 \kappa M m}{R^{3}}, \quad b=-\frac{\kappa M m}{R^{2}} .
$$


The motion of body 2 in the gravitational potential of body 1 is described by equation (Landau et al., 1965)

$$
m \ddot{r}=-\kappa \frac{M m}{r^{2}}+\frac{J^{2}}{m r^{3}},
$$

where $J$ is the angular momentum of body 2 . In the inerval $(-\delta, \delta)$ we can write

$$
r(t)=R+\eta(t)
$$

and using approximation

$$
\frac{1}{(R+\eta)^{2}} \approx \frac{1}{R^{2}}\left(1-\frac{2 \eta}{R}\right), \quad \frac{1}{(R+\eta)^{3}} \approx \frac{1}{R^{3}}\left(1-\frac{3 \eta}{R}\right)
$$

we get after insertion of eq. (6) into eq. (5):

$$
\ddot{\eta}+\omega^{2} \eta=\lambda
$$

where

$$
\begin{gathered}
\omega^{2}=\frac{3 J^{2}}{m^{2} R^{4}}-\frac{2 \kappa M}{R^{3}} \\
\lambda=\frac{J^{2}}{m^{2} R^{3}}-\frac{\kappa M}{R^{2}} .
\end{gathered}
$$

For the circle motion we have $J=m \omega R^{2}, r=R$ and from eqs. (9) and (10) it follows:

$$
\omega=R^{-3 / 2}(\kappa M)^{1 / 2} ; \quad \lambda=0 .
$$

It is easy to see that the solution of eq. (8) is of the form:

$$
\eta(t)=\Lambda \cos (\omega t+\vartheta)+\frac{\lambda}{\omega^{2}}
$$

where $\Lambda$ and $\vartheta$ are constants involving the initial conditions of motion of the body 2 .

So far we have supposed no dynamics of mediation of the interaction between body 1 and 2. However, only the model involving the mechanism of mediation of interaction can describe logically consistent reality and explain the Newton puzzle. Let us try to elaborate the consistent and realistic model which describes the mechanism of mediation.

\section{The string mediation of interaction}

In this section we will solve the motion of a body 2 at the end of the string on the assumption that the tension in the string is nonlinear and it generates the Newton law in the statical regime. We will give the rigorous mathematical formulation of such problem named the Newton-Pardy string mediation of interaction. While for the Hook tension the problem has solution by the Fourier method, in case of the nonlinear tension it is not possible to use this method.

There is no evidence about solution of this Newton-Pardy string problem in the textbooks of mathematical physics, or, in the mathematical journals. So, it seems, we solve this problem for the first time. 
Let be given the string, the left end of which is fixed at beginning and the right end is at point $l$ at the state of equilibrium. The deflection of the string element $d l$ at point $x$ and time $t$ let be $u(x, t)$ where $x \in(0, l)$ and

$$
\eta(t)=u(l, t), \quad \eta(0)=u(l, 0) .
$$

Then, the motion of body 2 is described by the motion of the right end-point of the string, when the left point is constantly fixed at the origin.

The differential equation of motion of string elements can be derived by the following way: We suppose that the force acting on the element $d l$ of the string is given by the law:

$$
T(x, t)=-E S\left(\frac{\partial u}{\partial x}\right)^{-2},
$$

where $E$ is the modulus of elasticity, $S$ is the cross section of the string. We easily derive that

$$
T(x+d x)-T(x)=2 E S\left(u_{x}\right)^{-3} u_{x x} d x .
$$

The mass $d m$ of the element $d l$ is $\varrho E S d x$, where $\varrho$ is the mass density of the string matter and the dynamical equilibrium gives

$$
\varrho S d x u_{t t}=2 E S u_{x x}\left(u_{x}\right)^{-3} d x .
$$

Putting

$$
\varrho=\varrho_{0} \frac{2}{\left(u_{x}\right)^{3}} ; \quad \varrho_{o}=\text { const. }
$$

we get

$$
\frac{1}{c^{2}} u_{t t}-u_{x x}=0 ; \quad c=\left(\frac{E}{\varrho_{0}}\right)^{1 / 2} .
$$

The last procedure was performed evidently in order to get the wave equation.

Now, let us look for the correspondence between the string tension and the Newton law. Putting $u_{t t}=0$ we get the stationary case with the solution

$$
u(x, t)=\alpha x+\beta .
$$

Because $u(0, t) \equiv 0$, we get $\beta=0$. Then $u_{x}(x, t)=\alpha$ is not dependent on $x$ and according to the definition of the tension the force is constant along the length of the string which is the same result as in the case with the Hook law.

For sufficiently big elongation we have $u(l) \gg l$ and the elongation at point $l$ is the distance of the right end of the string from the origin and it means that the force acting on the right end of the string is proportional to the minus square of the distance of the right end of the string as in the Newton gravitational law. So, we have demonstrated that the Newton gravitational force can be realized by the string, while the Newton original force involves no mediation between two bodies. Now, we can repeat the formulation of the problem dscribed in the previous section in such a way that we will use the dynamical equation (18) instead of eq. (5). So, let us approach the solution of the problem of the motion of body on the end of the string where the tension of the string is defined by equation (14). 
From (19) we have:

$$
\alpha=\frac{u(l, t)}{l} .
$$

Thus,

$$
T(l, t)=-\frac{E S l^{2}}{u^{2}(l, t)}=-\kappa \frac{m M}{u^{2}(l, t)},
$$

which gives the relation between the string constants and the gravitating parameters

$$
E S l^{2}=\kappa m M .
$$

The complete solution of eq. (18) includes the initial and boundary conditions. The simplest nontrivial initial conditions can be chosen with regard to the character of the problem and they are:

$$
u(x, 0)=\frac{R}{l} x, \quad u_{t}(x, 0)=0 .
$$

The boundary conditions are given with respect to the dynamical equation (5):

$$
u(0, t)=0, \quad m u_{t t}(l, t)=T(l, t)+\frac{J^{2}}{m u^{3}(l, t)} .
$$

The solution of the wave equation with the strongly nonlinear boundary conditions is evidently beyond the possibility of the present mathematical physics. Nor the Fourier method, nor the d'Alembert one can be used in solution of our problem. So we are forced to find only the approximation of this problem. For this goal we write:

$$
u(x, t)=\frac{R}{l} x+v(x, t),
$$

from which follows

$$
u_{x}(x, t)=\frac{R}{l}+v_{x}, \quad u(l, t)=R+v
$$

and we suppose that $v \ll R$. In such a way the intial conditions are:

$$
v(x, 0)=0, \quad v_{t}(x, 0)=0 .
$$

The approximative formulae are given in the following form:

$$
\begin{gathered}
\frac{1}{u_{x}^{2}(x, t)} \approx \frac{l^{2}}{R^{2}}-\frac{2 v_{x} l^{3}}{R^{3}}, \\
\frac{1}{u^{3}(x, t)} \approx \frac{1}{R^{3}}-\frac{3 v}{R^{4}} .
\end{gathered}
$$

So, we get the new problem of mathematical physics: the wave equation

$$
v_{t t}=c^{2} v_{x x}
$$

with the initial conditions

$$
v(x, 0)=0 ; \quad v_{t}(x, 0)=0
$$


and with the boundary conditions

$$
v(0, t)=0 ; \quad m v_{t t}(l, t)=a+b v_{x}(l, t)+d v(l, t),
$$

where we have put

$$
a=-\kappa \frac{M m}{R^{2}}+\frac{J^{2}}{m R^{3}} ; \quad b=\frac{2 \kappa M m}{R^{3}} l ; \quad d=-\frac{3 J^{2}}{m R^{4}} .
$$

The equation (30) with the initial and boundary conditions (31) and (32) represents one of the standard problems of the mathematical physics and can be easily solved using the Laplace transform (Arfken, 1967):

$$
\hat{L} u(x, t) \stackrel{d}{=} \int_{0}^{\infty} e^{-p t} u(x, t) d t \stackrel{d}{=} u(x, p) .
$$

Using (30) we get with $\hat{L} v(x, t) \stackrel{d}{=} \varphi(x, p)$ :

$$
\begin{gathered}
\hat{L} v_{t t}(x, t)=p^{2} \varphi(x, p)-p v(x, 0)-v_{t}(x, 0)=p^{2} \varphi(x, p) \\
\hat{L} v_{x x}(x, t)=\varphi_{x x}(x, p) ; \quad \hat{L} a=\frac{a}{p} ; \quad \hat{L} v(0, t)=\varphi(0, p)=0 .
\end{gathered}
$$

After elementary mathematical operations we get the differential equation for $\varphi$ in the form:

$$
\varphi_{x x}(x, p)-k^{2} \varphi(x, p)=0 ; \quad k=p / c .
$$

with the boundary condition in eq. (36).

We are looking for the the solution of eq. (37) in the form

$$
\varphi(x, p)=c_{1} \cosh k x+c_{2} \sinh k x .
$$

We get from the boundary conditions in eq. (36) $c_{1}=0$ and

$$
c_{2}=\frac{a}{p} \frac{1}{\left(m p^{2}-d\right) \sinh k l-b k \cosh k l} .
$$

The corresponding $\varphi(x, p)$ is of the form:

$$
\varphi(x, p)=\frac{a}{p} \frac{\sinh k x}{\left(m p^{2}-d\right) \sinh k l-b k \cosh k l}
$$

The corresponding function $v(x, t)$ follows from the theory of the Laplace transform as the mathematical formula:

$$
\begin{gathered}
v(x, t)=\frac{1}{2 \pi i} \oint e^{p t} \varphi(x, p) d p=\sum_{p=p_{n}} r e s e^{p t} \varphi(x, p)= \\
\sum_{p=p_{n}} \operatorname{reses}^{p t} \frac{\sinh k x}{p} \frac{\sin }{\left(m p^{2}-d\right) \sinh k l-b k \cosh k l},
\end{gathered}
$$

where $p_{n}$ are poles of the function $\varphi(x, p)$ and they are evidently given by equation

$$
\left[\left(m p^{2}-d\right) \sinh k l-b k \cosh k l\right]=0,
$$


which is equivalent with $k \rightarrow i k$ to

$$
\tan k l=\frac{-b k}{m c^{2} k^{2}+d} .
$$

In case of $k \ll 1$ we have two solutions: $p_{0}=0$ and

$$
p_{1 / 2}= \pm\left(\frac{3 J^{2}}{m^{2} R^{4}}-\frac{2 \kappa M}{R^{3}}\right)^{1 / 2}
$$

which is in agreement with eq. (9) obtained by the approximation of classical Kepler problem. Further we have got the oscillations with frequences $p_{n}$ in the higher order approximation:

$$
p_{n} \rightarrow \frac{n \pi}{l}, \quad n \gg 1
$$

At present time it is still question, how to detect these oscillations, or, if it is possible to use the experimental procedures of Braginskii et al. (1977) for the detection. The analogous situation was in quantum physics, where the zero frequences of vacuum was considered as meaningless till it was shown by Casimir that they give the atractive force between two conductive plates. It is not excluded that the Zitterbewegung of cellestial bodies will be confirmed by NASA laser experiments.

\section{Discussion}

The basic heuristical idea of this article was the string realization of the gravitational force between two bodies.

In order to realize this idea we introduced the string of the length $l$ with the nonlinear tension which generates in the statical situation the Newton law at the distances much greater then is the fundamental length of the string. We have solved this problem only approximately because at present time the exact solution is beyond possibilities of mathematics.

While the string with the Hook tension has the equilibrium state, our string is in the dynamical state.

The difficulties with the action-at-distance as is an indication of the limitation of the Newtonian theory. The general relativity solves the problem as the metric theory of the gravitational interaction. General relativity is the geometry theory of space time and as such it is the physical model based on the Riemann and Gauss ideas. In General relativity, there is the fundamental notion, the metric tensor, while in the Newton theory the basic building stone is force. In our theory, which is the dynamical version of the Hook theory of string is the basic notion the tension of the string.

The string is between any two masses and it means that universe is occupied by strings, vacuum and by bodies and particles. Our planet is for instance connected by strings with all stars in universe.

Our problem was never defined to our knowledge in the mathematical or physical textbooks, monographies or scientific journals. Thus, our approach is original.

The proposed model can be also related in the modified form to the problem of the radial motion of quarks bound by a string and used to calculate the excited states of such system. The original solution was considered by Bardeen et al. (1976; 1976) Chodos et al. (1974) and by Frampton (1975). The new analysis of such problem was performed by 
Nesterenko (1990) and author (Pardy, 2016). So, there are open way in particle physics to follow our approach. It is not excluded that the Zitterbewegung of moon will be confirmed by NASA laser systems (NASA first!).

\section{References}

Arfken, G. Mathematical Methods for Physicists (Academic Press, New York and London, 1967).

Bardeen, W. Bars, A. I., Hanson, A. J. and Peccei, R. D. (1976). Quantum Poincare' covariance of the two-dimensional string, Phys. Rev. D 13, 2364.; ibid. 14, 2193.

Bentley, R. Works of Richard Bentley, Vol. 3, pp. 210-211,; Letter of Newton to Bentley, Trinity College, Jan 17, 1692-1693.

Braginsky, V. B., Caves, C. M. and Thorne, K. S. (1977). Laboratory experiments to test relativistic gravity, Phys. Rev. D 15, 2047.

Chodos, A. and Thorn, C. B. (1974). Making the massless string massive, Nucl. Phys. 72,509 .

Frampton, P. (1975). String approaches to hadron structure, Phys. Rev. D 12, 538.

Landau, L. D. and Lifschitz, E. M. Mechanics (Nauka, Moscow, 1965). (in Russian).

Newton, I. Principia Mathematica (University of California Press, Berkeley and Loss Angeles, 1966). p. 634.

Nesterenko, V. V. (1990). On the radial motion of quarks bound by a string, Z. Phys. C - Particles and Fields 47, 111.

Pardy, M. (1980). The string model of action at a distance, University of J. E. Purkynje (UJEP), Unpublished.

Pardy, M. (1996). The string model of gravity, arXiv:gr-qc/9602007v1, 5 Feb. 1996.

Pardy, (2016). The excited mesons due to laser pulse, Intellectual Archive, 2016-09-26, ID 1764.

Tikhonov, A. N. and Samarskii, A. A. The Equations of Mathematical Physics (Nauka, Moscow, 1977). (in Russsian).

Stanford Encyclopedia of Philosophy, (2006), Newton's Philosophy. 